\title{
The chronometry of risk processing in the human cortex
}

\section{Mkael Symmonds ${ }^{1,2}{ }^{*}$, Rosalyn J. Moran ${ }^{1,3}$, Nicholas D. Wright ${ }^{1}$, Peter Bossaerts ${ }^{4,5}$, Gareth Barnes ${ }^{1}$ and Raymond J. Dolan ${ }^{1}$}

1 Wellcome Trust Centre for Neuroimaging, Institute of Neurology, University College London, London, UK

${ }^{2}$ Nuffield Department of Clinical Neurosciences, Oxford University, John Radcliffe Hospital, Headington, Oxford, UK

${ }^{3}$ Virginia Tech Carillion Research Institute, Virginia Tech, Roanoke, VA, USA

${ }^{4}$ Caltech Laboratory for Experimental Finance, California Institute of Technology, Pasedena, CA, USA

${ }^{5}$ Swiss Finance Institute, Ecole Polytechnique Federale Lausanne, Lausanne, Switzerland

\section{Edited by:}

Aldo Rustichini, University of

Minnesota, USA

Reviewed by:

Paul Sajda, Columbia University, USA

V. S. Chandrasekhar Pammi, University of Allahabad, India

\section{*Correspondence:}

Mkael Symmonds, Nuffield Department of Clinical

Neurosciences, Oxford University,

John Radcliffe Hospital, West Wing,

Level 6, Oxford OX3 9DU, UK

e-mail:mkael.symmonds@

ndcn.ox.ac.uk
The neuroscience of human decision-making has focused on localizing brain activity correlating with decision variables and choice, most commonly using functional MRI (fMRI). Poor temporal resolution means these studies are agnostic in relation to how decisions unfold in time. Consequently, here we address the temporal evolution of neural activity related to encoding of risk using magnetoencephalography (MEG), and show modulations of electromagnetic power in posterior parietal and dorsomedial prefrontal cortex (DMPFC) which scale with both variance and skewness in a lottery, detectable within $500 \mathrm{~ms}$ following stimulus presentation. Electromagnetic responses in somatosensory cortex following this risk encoding predict subsequent choices. Furthermore, within anterior insula we observed early and late effects of subject-specific risk preferences, suggestive of a role in both risk assessment and risk anticipation during choice. The observation that cortical activity tracks specific and independent components of risk from early time-points in a decision-making task supports the hypothesis that specialized brain circuitry underpins risk perception.

Keywords: decision-making, magnetoencephalography (MEG), risk, neuroeconomics, cortex

\section{INTRODUCTION}

Risk describes uncertain scenarios wherein chosen actions yield a range of possible outcomes that are quantified by different statistical features in a distribution. On the one hand variance measures outcome spread (uncertainty). On the other hand skewness measures asymmetry, where positive skewness describes distributions with occasional returns well-above average (e.g., casino gambles with high potential winnings) and negative skewness describes distributions with occasional poor outcomes (e.g., rare catastrophic occurrences during routine surgery) (Coombs, 1960; Jullien and Salanie, 2000). Trading off these distinct aspects of risk against potential returns is a central component of valuebased choice (Coombs, 1960; Weber and Johnson, 2008), making it likely that evolution has endowed specialized mechanisms for this evaluation.

Considerable evidence indicates involvement of specific cortical and subcortical neural regions in decision-making under risk, ranging from electrophysiological studies in animals (e.g., Fiorillo et al., 2003), lesion-based and neuromodulatory studies (e.g., Knoch et al., 2006; Clark et al., 2008; St. Onge and Floresco, 2010) to neuroimaging investigations in humans with positron emission tomography (PET) (e.g., Ernst et al., 2002), and fMRI (e.g., Critchley et al., 2001; Kuhnen and Knutson, 2005; McCoy and Platt, 2005; Preuschoff et al., 2006; Tobler et al., 2007; Christopoulos et al., 2009; Venkatraman et al., 2009; Mohr et al., 2010b). Recently, several fMRI studies have highlighted scaled responses to different components of statistical risk in particular in posterior parietal and prefrontal cortices (Huettel et al., 2005;
Smith et al., 2009; Xue et al., 2009; Symmonds et al., 2010, 2011; Bach and Dolan, 2012). In contrast to previous electroencephalographic or MEG studies of economic decision-making focused on the response to reward feedback (e.g., Gehring and Willoughby, 2002; Hewig et al., 2007), here we investigated the evaluation stage of an economic decision. We capitalize on the temporal fidelity of MEG to study the chronometry of risk responses within these identified parietal and prefrontal regions at a sub-second timescale.

While fMRI allows precise localization of risk-sensitive networks, poor temporal resolution (Kim et al., 1997) limits its ability to inform the temporal sequence of risk appraisal. This is an important lacuna, as risky choices can be evaluated within 1-3 s (Kuhnen and Knutson, 2005; Huettel et al., 2006; Xue et al., 2009), which mandates rapid neural processing of salient statistical features prior to response generation. Moreover, neural responses to risk observed with fMRI might represent an initial encoding of statistical risk or alternatively may represent a risk anticipation signal after option evaluation and any motor preparation signal. To disambiguate these two possibilities we aimed to first test whether it was possible to detect magnetoencephalographic signal changes correlating with risk in the initial evaluation phase of a decision-making task, and secondly to demonstrate whether these signals are detected prior to, or following the formation of signals correlating with an individual's choices. If risk perception is supported by specialized cortical representation, leading to option evaluation and choice, we expect early variance and skewness processing before or concurrent with 
signal changes predictive of choice. We hypothesized that these signals would be evident in already identified parietal and prefrontal candidate regions. Moreover, an influence of individual risk preference on choice-related signals has also been reported in anterior insula (Christopoulos et al., 2009; Tobler et al., 2009). An early effect of subjective preferences in insula would support an integral role in risk evaluation (Preuschoff et al., 2006), while a late effect would corroborate the alternative theory that insula is involved in an affective anticipation of a risky choice (Kuhnen and Knutson, 2005).

Here we show early variance and skewness cortical responses within parietal and prefrontal candidate regions, as well as preference-dependent activity in anterior insula. Thus, our findings suggest that specialized brain circuitry underpins rapid perception of discrete aspects of risk.

\section{MATERIALS AND METHODS PARTICIPANTS}

The study was approved by the Institute of Neurology (University College London) Ethics Committee. Seventeen subjects (mean age: 31 ; age range: $25-50$; 5 male) were recruited for the experiment. One (female) subject was excluded because of metal artifact due to dental work, and 1 (male) subject excluded because of excessive drowsiness and failure to make button-press responses during the experiment.

\section{TASK}

To dissociate different components of risk, in terms of dispersion (variance) and asymmetry of outcomes (skewness), we adapted a previously described decision-making task (Symmonds et al., 2011) that controlled for the distribution of possible outcomes. The task, by design, ensured variance and skewness of a set of lotteries could be manipulated independently. Consequently, variance and skewness of gambles were orthogonal factors and this enabled us to test whether neural activity evoked by variance could be segregated in time and place from that evoked by skewness.

Participants were required to choose between taking a "sure" (fixed) amount of money or elect to "gamble" (choosing to play a lottery with four potential outcomes). Gambles were represented as pie-charts, where variance and skewness of outcomes varied over a range, while the expected value of gambles was kept constant (Figures 1A,B). On each trial, we recorded choices and simultaneous neural responses (i.e. MEG signal changes) as a function of these changing variables, which allowed us to then determine both when, and where, within a priori regions of interest risk signals are represented.

\section{INDEPENDENT MANIPULATION OF VARIANCE AND SKEWNESS}

We constructed a stimulus set of 252 lotteries (each presented once) where variance and skewness were independent and varied over a range. For every level of variance (16 levels), we independently varied skewness (16 levels, 8 positively skewed, 8 negatively skewed). The expected value of the lotteries was kept constant (between 5.95 and $\mathfrak{E} 6.05$ ), and the sure amount alternative remained constant throughout at $\mathfrak{E} 4.50$. Stimuli were constrained to have 4 outcomes (segments of the pie chart), with outcome probabilities varying in minimum 0.1 increments between 0 and 1 so as to mitigate against probability distortion effects at small probabilities. These restrictions allow the generation of a space of possible lotteries varying in skewness and variance (Figure 1C). Using lotteries with multiple outcomes is crucial in enabling such a dissociation of risk dimensions and consequently we used 4 outcome lotteries throughout to control for perceptual and cognitive variability in task processing.

\section{PAYMENT}

At the end of the experiment, three trials were randomly selected and these were then played out for real. If subjects had elected to gamble, we resolved the lottery by an on-screen graphic of a ball spinning around the outside of the pie until it stopped at a randomly selected position. This procedure was also shown in the practice, to demonstrate the idea that the size of each segment of the pie chart represented the chance of that outcome occurring. Monetary earnings ranged between 13 and $\mathfrak{E} 35$ (mean $\mathfrak{E} 21.17$ ).

\section{BEHAVIORAL MODELING}

To demonstrate that participants had a behavioral sensitivity to our manipulated risk dimensions of variance and skewness, we compared behavioral models of risk evaluation.

For a given lottery with 4 potential outcomes $\left(m_{1}, m_{2}, m_{3}\right.$, $\left.m_{4}\right)$, with probabilities $p=p_{1}, p_{2}, p_{3}, p_{4}$, we define the statistical moments [expected value (EV), variance (Var), skewness (Skw)] of the outcome distribution as:

$$
\begin{aligned}
& E V=\sum_{n=1}^{4} m_{n} p_{n} \\
& V a r=\sum_{n=1}^{4}\left(m_{n}-E V\right)^{2} p_{n} \\
& S k w=\sum_{n=1}^{4}\left(m_{n}-E V\right)^{3} p_{n}
\end{aligned}
$$

In this study, we used the raw statistical moments to define variance and skewness. This use derives from the fact that any utility function can be approximated by preferences for summary statistics using a polynomial expansion (Scott and Horvath, 1980; Müller and Machina, 1987; D'Acremont and Bossaerts, 2008). In practice, the raw and normalized moments are correlated, and our focus here was to draw a distinction between responses to the spread (variance) and asymmetry (skewness) of outcomes, rather than systematically test neural signatures of alternative measures of these statistics.

We analyzed choice data by fitting a linear mean-varianceskewness model (MVS) where individuals are allowed to express different preferences for variance and skewness. To demonstrate sensitivity to both variables of interest, we compared the behavioral fit of this model to two alternatives; a model based on mean difference $(\mathrm{M})$ alone (where subjects only take account of the difference between the sure amount and the expected value of the gamble in selecting actions), and a mean-variance model (MV). 

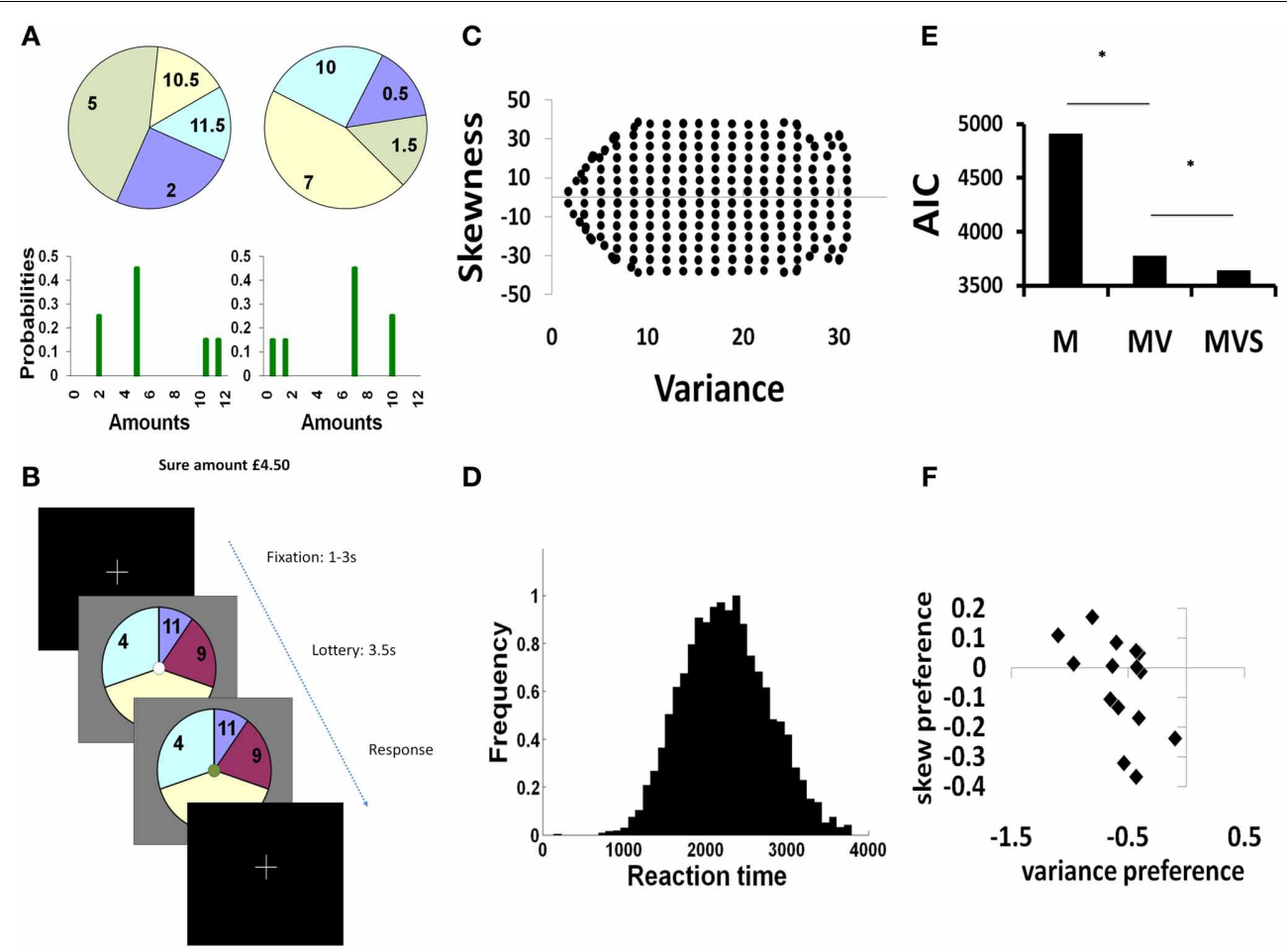

FIGURE 1 | Experimental paradigm and behavior. (A) We represented gambles on-screen as pie-charts. The pie chart was divided into four different segments showing possible outcomes from the lottery. The numbers written in each segment show the monetary value of each outcome (in pounds sterling) and the angle subtended by each segment indicated the probability of each outcome occurring. A positively skewed gamble (left) has a small chance of a better than average outcome (the tail of the distribution is to the right). Conversely, a negatively skewed gamble (right) has a small chance of a worse than average outcome (the tail is to the left). Both example gambles have identical variance and expected value.

(B) Each trial was self paced, with subjects first shown a fixation cross, and then pressing a button to commence the trial. Following initial button press, a pie chart gamble was presented for $3.5 \mathrm{~s}$, during which time the subject was required to make a two alternative forced choice between opting to gamble, or to take a sure amount of money of $£ 4.50$. Subjects selected actions by a button press, which was indicated on screen by a color change in the central fixation circle. At the end of the experiment, three trials were randomly selected and played out for real. If subjects had elected to gamble, we resolved the lottery by an on-screen graphic of a red ball spinning around the outside of the pie which stopped at a randomly selected position. (C) Plot of the stimulus space used in this study, showing 252 gambles independent in variance and skewness. The non-uniformity at the extremes of variance is limited by the restrictions on stimulus generation (a fixed expected value of 5.95-6.05f, 4 segment pie charts with a minimum probability of 0.1 and probability increments of 0.1 ). (D) Reaction times were normally distributed between $\sim 1-3 \mathrm{~s}$. $99.7 \%$ of button press responses occurred after $1 \mathrm{~s}$ following stimulus presentation. (E) Summed AIC scores for 3 models: mean only (M), mean-variance (MV), mean-variance-skewness (MVS). A lower score indicates a better model fit. The MV model was significantly better than the M model $\left(\chi^{2}:{ }^{*} p<1 \times 10^{-5}\right)$, while the MVS model was significantly better than MV $\left(x^{2}:{ }^{*} p<1 \times 10^{-5}\right)$. (F). Parameter estimates from the MVS model reveal a range of preferences for variance (negative coefficient reflects variance aversion), and skewness (coefficient reflects preference for positive vs. negative skewness).
We define the subjective value, or utility (U) of each lottery for our models:

Mean model (M)

$$
U=E V
$$

Mean-variance model (MV)

$$
U=E V+\rho \operatorname{Var}
$$

Mean-variance-skewness model (MVS)

$$
U=E V+\rho \operatorname{Var}+\lambda S k w
$$

$\rho$ and $\lambda$ are free parameters, $\rho$ reflecting preference for variance, and $\lambda$ reflecting preference for positive vs. negative skewness, respectively.
Our models compare the utility of the lottery with the value of the sure amount $(S)$ to generate a trial-by-trial probability of choosing the lottery over the sure amount, using a logistic/softmax function which allows for noise in action selection (by free parameter $\beta$ ).

$$
P_{\text {choose gamble }}=\frac{1}{1+\exp (-(1 / \beta)(U-S))}
$$

We estimated best-fitting model parameters using maximum likelihood analysis, with optimization implemented with a non-linear Nelder-Mead simplex search algorithm in Matlab (Matlab, Natwick, USA). We compared models using the Akaike Information Criterion (AIC), a comparison which penalizes model complexity (Akaike, 1974). 


\section{MEG-EXPERIMENTAL SETUP, RECORDING PARAMETERS AND PREPROCESSING}

We recorded MEG continuously (sample rate: $1200 \mathrm{~Hz}$ ), using a 274-channel whole-head system (CTF Omega), with participants in a seated position. Stimuli were presented and responses recorded using Cogent presentation software (Wellcome Trust Centre for Neuroimaging, London) written in MATLAB (version 6.5.1, MathWork, Natick, MA). Synchronization of MEG data with the stimulus train was ensured by writing simultaneous timing triggers on each trial to an MEG data channel using the Cogent software (outportb command). Visual cues were projected onto a screen directly in front of the participant. Choices were indicated by pressing a button box with the right index finger. Imaging data were analyzed using Statistical Parametic Mapping software (routines in the academic freeware package SPM8; Wellcome Trust Centre for Neuroimaging, UK, www.fil. ion.ucl.ac.uk/spm).

MEG data were epoched to obtain $1000 \mathrm{~ms}$ data segments corresponding to the first second after presentation of the stimulus. This cutoff was chosen as the time window during which stimuli were being evaluated and before button press responses were emitted. On $99.7 \%$ of trials motor responses occurred only after this point (Figure 1D). Data were downsampled to $200 \mathrm{~Hz}$, bandpass filtered from 1 to $80 \mathrm{~Hz}$, and baseline corrected. One hundred milliseconds of MEG data prior to presentation of stimulus (when fixation cross was on screen) was sampled as a baseline period.

We performed artifact rejection using an algorithm that rejected all trials where the root mean square (RMS) power was a factor of 10 greater than the average RMS power per trial across subjects.

\section{MEG-SOURCE LEVEL ANALYSIS}

We reduced the dimensionality of our analysis by focusing on four predefined time windows (0-250 ms, 250-500 ms, 500-750 ms, $750-1000 \mathrm{~ms})$ and four frequency bands theta $(4-8 \mathrm{~Hz})$, alpha $(8-16 \mathrm{~Hz})$, beta $(16-32 \mathrm{~Hz})$ and gamma $(32-64 \mathrm{~Hz})$, using the multiple sparse prior routine within SPM8 (Friston et al., 2008), with group constraints (Litvak and Friston, 2008). This inverse solution performs an iterative Bayesian optimization to estimate current density on a cortical surface template mesh of several hundred patches, where the mesh is a tessellated template based on the canonical Montreal Neurological Institute (MNI) brain (Mazziotta, 2001), with a single shell template head model. We selected a $250 \mathrm{~ms}$ temporal window length given we examine oscillatory responses from 4 to $48 \mathrm{~Hz}$ (i.e., minimum one cycle length). Hence we obtained 16 ( 4 time $\times 4$ frequency) source images per subject per trial.

Our contrasts of interest pertained to our 3 parametric variables of variance, skewness and subject's choice (a categorical variable indicating gamble or sure choice) on each trial. We implemented a standard hierarchical analysis, first estimating within-subjects effect sizes for each of these 3 parametric variables in a general linear model. For each subject, source-localized data were entered into a multiple linear regression against predictor variables corresponding to level of variance, skewness and choice on each trial. All variables were normalized to the range $0-1$ and mean-centered. This regression analysis was performed at each of the above time and frequency bins, generating withinsubject statistical maps of the regression coefficients corresponding to variance, skewness and choice variables. To make group (between-subject) inferences we then entered each of these 16 (4 time $\times 4$ frequency) source level statistical images per subject into a two-factor repeated measures ANOVA. This second-level ANOVA also included indicator regressors to account for subject effects. Separate ANOVAs were constructed to analyse effects of variance, skewness and choice regressors, respectively.

We were agnostic about the direction of any correlation between decision variables and MEG responses and had no a priori assumptions about the frequency bands in which specific effects would be expressed. For inference, $F$-tests were performed to isolate the main effect over time, collapsing across frequency bands. In other words, we ask where source-localized power correlates with variance, skewness or choice at any of our 4 time windows. Rather than a broadband analysis, we enter trial-bytrial source localized power corresponding to each of our 4 pre-specified frequency bands $(4-8,8-16,16-32,32-64 \mathrm{~Hz})$. We analyzed source level induced responses in these windows over subjects using a second-level ANOVA. We used an omnibus test as we had no a priori assumptions about the frequency bands at which effects could be expressed and to avoid multiple comparisons. Note the $F$ test denominator degrees of freedom is derived from the dimensions of the second level design matrix, with 15 (subject) $\times 4$ (time bands) $\times 4$ (frequency bands) rows. Subsequent post-hoc $t$-tests were then used to delineate the effect size within each time-bin only in areas expressing a significant main effect.

\section{STATISTICAL REPORTING AND FIGURES}

We restricted our tests to likely cortical regions expressing responses to statistical risk during decision-making, guided by a priori knowledge regarding localization of a risk evaluation network. A meta-analysis of functional imaging studies has identified consistent decision risk (i.e., risk processing before or during choice) responses across several studies in dorsomedial prefrontal cortex (DMPFC), anterior insula and parietal cortices (Mohr et al., 2010a), regions also identified in our similarly designed study where variance and skewness were independently manipulated (Symmonds et al., 2011). We therefore draw inferences in source space by mapping observed profiles of responses in a mask of 6 regions - bilateral posterior parietal, dorsomedial prefrontal, and anterior insular cortices. These were defined using anatomical labels in WFU PickAtlas v2.5 (Maldjian et al., 2003), a software method for generating region of interest masks based on the Talairach Daemon database, converted into MNI spatial coordinates (Lancaster et al., 1997, 2000). We report voxel-wise significant results at a $p<0.005$ threshold. Brain image figures show second-level SPM-F maps, superimposed upon a canonical brain image, thresholded at $p=0.01$. Stereotactic coordinates are reported in MNI space (Mazziotta, 2001). Bar plots show effect sizes across time within regions showing a significant main effect.

\section{RESULTS}

Fifteen subjects chose between gambling on a lottery or selecting a sure amount of money in a set of individually presented 
lotteries where we independently manipulated variance and skewness. On each trial, we recorded choices and simultaneous neural responses (i.e., MEG signal changes) as a function of these changing variables (Figures 1A,B), which allowed us to then determine both when, and where, within a priori regions of interest risk signals are represented. To demonstrate behavioral sensitivity to both variance and skewness, we measured subjective preferences for both these variables using behavioral economic models. We analyzed activity unfolding from stimulus presentation, allowing us to map the temporal sequence of events underlying the initial stage of risk information processing.

\section{BEHAVIOR}

Our stimulus set (Figure 1C) was designed such that participants would evenly distribute their choices between gamble and sure amounts. This approach maximizes power for both behavioral fitting and subsequent analysis of MEG activity corresponding to choice. As planned, our subjects on average distributed their choices between gamble and sure options throughout the course of the experiment (mean percentage of gamble choices $=55 \%$, std. $17 \%)$. There were few error (missed) trials ( $0.4 \%$ of all trials). Mean response times (RTs) (from stimulus presentation to button press) were $2.26 \pm(\mathrm{SD}) 0.53 \mathrm{~s}$ (Figure 1D).

In designing the paradigm, we aimed to minimize eye movement, and render this uncorrelated with variables of interest. Thus, subjects were required to fixate on a central fixation cross, and pie chart lottery stimuli of the same size were centrally presented with random orientation on the screen. Each lottery had 4 outcomes with numerical amounts placed in identically spaced radial locations (Figure 1A), to ensure that an equal amount of eye movement per trial was required to take in the statistical information, such that saccades would average out across trials and subjects.

We further checked whether eye movements were correlated with variables of interest, recording electro-oculogram (EOG) data (with 3 EOG electrodes placed above, below and lateral to the left eye), in 10 of our participants. We measured eye movement per trial by the magnitude of signal change (from mean) across the EOG channels. The total amount of eye movement in our $1000 \mathrm{~ms}$ post-stimulus window of interest was not significantly correlated with the lottery statistics [variance: $t_{(1,9)}=1.2$, $p=0.27$; skewness: $t_{(1,9)}=1.4, p=0.19$ ] or the subject's choice $\left[t_{(1,9)}=0.9, p=0.37\right]$.

\section{Sensitivity to both variance and skewness}

Individuals' choices were sensitive to both variance and skewness. To formally test this sensitivity we compared a mean-varianceskewness model (MVS), where individuals are allowed to express preferences for both variance and skewness, to a set of alternative decision models (see Materials and Methods). As predicted, a mean-variance-skewness (MVS) model provided a significantly better fit to the observed behavioral data than the alternatives (summed AIC scores: M: 4909; MV: 3778; MVS: 3642); MVS model posterior probability $>0.99$ (very strong evidence in favor of MVS) (Figure 1E). Our winning MVS model provided subjectspecific preference metrics for variance and skewness (Figure 1F). All subjects were averse to variance (average variance preference:
$-0.56 \pm \mathrm{SD} 0.25$ ), and 7/15 preferred negative to positive skewness (average skewness preference: $-0.06 \pm$ SD 0.16). Beta (temperature) values for the logistic function were low, indicating that choices were well-partitioned by the linear model (average beta $=$ 0.13 ; SD 0.06).

\section{MEG SOURCE LEVEL ANALYSIS}

We report induced time-frequency responses where our dependent variable is trial-by-trial oscillatory power induced by specific components of a decision. This is in contrast to previous studies using electrographic or magneto-encephalographic recording of economic decision making (Gehring and Willoughby, 2002; Schutter et al., 2004; Hewig et al., 2007; Hedgcock et al., 2010; Harris et al., 2011; Steffen et al., 2011; Yu et al., 2011), which often focus on evoked responses (event-related potentials-ERPs, or fields-ERFs), usually to feedback about decision outcome. While receipt of a reward or loss can generate synchronized eventrelated signals occurring rapidly following feedback, we focused on time-frequency responses as we were interested in risk evaluation signals evolving over an extended period of hundreds of milliseconds, with considerable potential variability which may curtail detection in evoked responses given signal changes might cancel out in the average.

\section{Early encoding of variance and skewness}

Our first goal was to test whether we could detect magnetoencephalographic signal changes corresponding to different aspects of risk, and hence characterize this temporal processing during decision-making. We localized induced power in four prespecified time windows (0-250, 250-500, 500-750, 750-1000 ms) following stimulus presentation, at each of these 4 frequency windows [4-8 Hz ("theta"), 8-16 Hz ("alpha"), 16-32 Hz ("beta"), $32-48 \mathrm{~Hz}$ ("gamma”)]. We report results within masked regions of interests to spatially constrain the analyses. These comprise parietal, prefrontal and insula cortex, based on previous investigations (see Materials and Methods).

We observed an early effect of variance in left posterior parietal cortex $\left[F_{(3,210)}=4.60, p=0.004\right.$; Figure $\left.2 \mathrm{~A}\right]$. This linear modulation of induced power commenced in the initial $0-250 \mathrm{~ms}$ following lottery presentation $\left[t_{(1,210)}=2.32, p=\right.$ $0.011]$, peaking between 250 and $500 \mathrm{~ms}\left[t_{(1,210)}=2.74, p=\right.$ 0.003] (Figure 2B). On presentation of high (vs. low) variance gambles, power $(8-32 \mathrm{~Hz})$ initially decreased between 0 and $250 \mathrm{~ms}$ [peak effect: $16-32 \mathrm{~Hz}, t_{(1,210)}=2.06, p=0.020$ ], before a broadband increase in power between 250 and $500 \mathrm{~ms}$ [peak effect: $4-8 \mathrm{~Hz}, t_{(1,210)}=1.75, p=0.040$ ] (Figure 3).

Gamble skewness significantly correlated with MEG signal power in DMPFC, with a peak effect seen on the right [R DMPFC: $F_{(3,210)}=4.76, p=0.003$; Figure $2 \mathrm{C}$ ], a response present between 250 and $750 \mathrm{~ms}\left[t_{(1,210)}=4.21, p<0.001\right]$ (Figure 2D). Within the area of peak effect there was significantly greater power during presentation of negative relative to positively skewed gambles driven by increased alpha $(8-16 \mathrm{~Hz})$ and gamma $(32-48 \mathrm{~Hz})$ power at $500-750 \mathrm{~ms}$. Thus, the earliest responses to variance are observed $250 \mathrm{~ms}$ before the responses to skewness (Figure 4), although there is substantial overlap between the two signals. 


\section{A Variance}
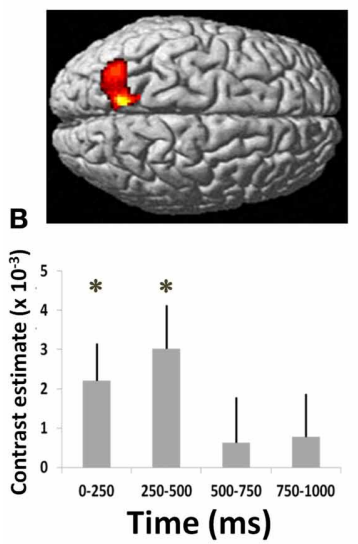

c Skewness
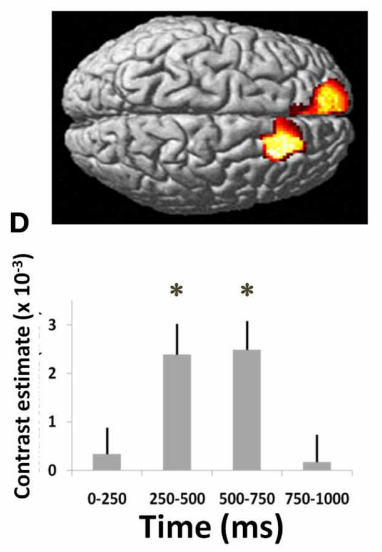

E Choice
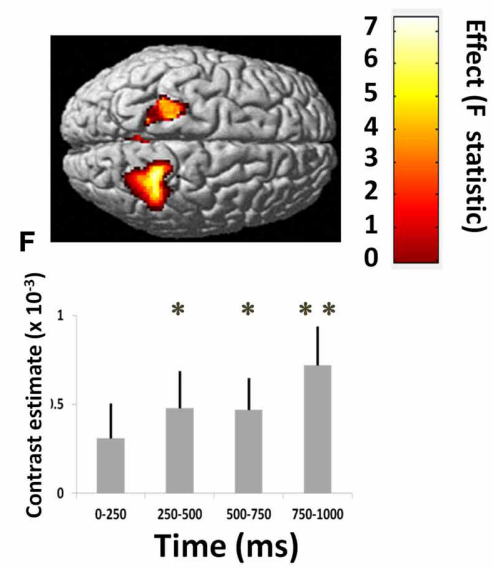

FIGURE 2 | Responses to risk and choice. Results of group analysis GLM (two-factor repeated measures ANOVA) of signal power correlating with variance, skewness, and subject's choice. Figures show

source-reconstructed induced power parametrically correlating with each of these three regressors. GLM-estimated coefficients for each parametric variable give the estimated slope of the best linear fit, where the null hypothesis at each source is that activity is insensitive to variance, skewness or choice (i.e., the regression coefficient equals zero). Figures show second-level SPM-F image thresholded at $p<0.01$, superimposed upon a canonical brain $\left({ }^{*} p<0.01,{ }^{* *} p<0.0001\right.$; colorbars show F-statistics). $T=0$ corresponds to time of stimulus presentation. (A) Power correlation with variance in left posterior parietal cortex [all effects across 4 frequency bands: "theta" $(4-8 \mathrm{~Hz})$, "alpha" $(8-16 \mathrm{~Hz})$, "beta" $(16-32 \mathrm{~Hz})$, "gamma" (32-48 Hz)]. (B) Timecourse of effect, showing significant effects in first $0-250 \mathrm{~ms}$ window, peaking at $250-500 \mathrm{~ms}$ (variance peak voxel at $-12,-56,52)$. (C) Power correlation with skewness in left and right DMPFC (across all frequencies). (D) Timecourses of effects in right DMPFC (skewness peak voxel at 22, 24, 34). (E) Power correlation with trial-by-trial choices (gamble vs. sure, across all frequency bands). The effect is seen bilaterally posterior to the central sulcus. (F) Timecourse for peak voxel (at 28, -46, 50) shows effects commencing at 250-500 ms, maximal at $750-1000 \mathrm{~ms}$ after stimulus presentation.

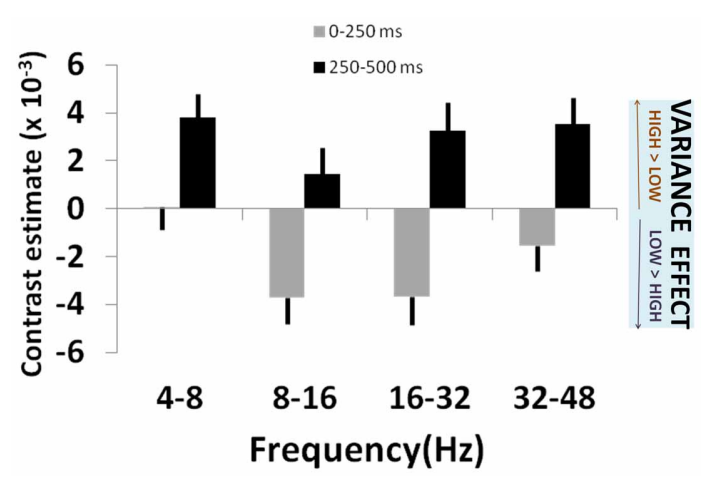

FIGURE 3 | Variance effects across frequency bands. Plot of effect sizes for correlation of signal power with lottery variance at 0-250 ms (gray bars) and $250-500 \mathrm{~ms}$ (black bars) time windows, for each of 4 specified frequency bands. This corresponds to times at which significant main effects of variance (i.e., across all frequencies) were observed (see Figure 2). Positive contrast estimates reflect greater signal power for high risk (high variance) than low risk (low variance) lotteries. See main text for details and statistical reporting.
These data show that both components of risk (variance and skewness) induce independent scaled modulations of oscillatory activity at similar time epochs in parietal (variance) and prefrontal cortices (skewness). These are regions previously identified as candidate anatomical loci using fMRI, and the MEG data now reveal that these responses occur very rapidly following stimulus presentation.

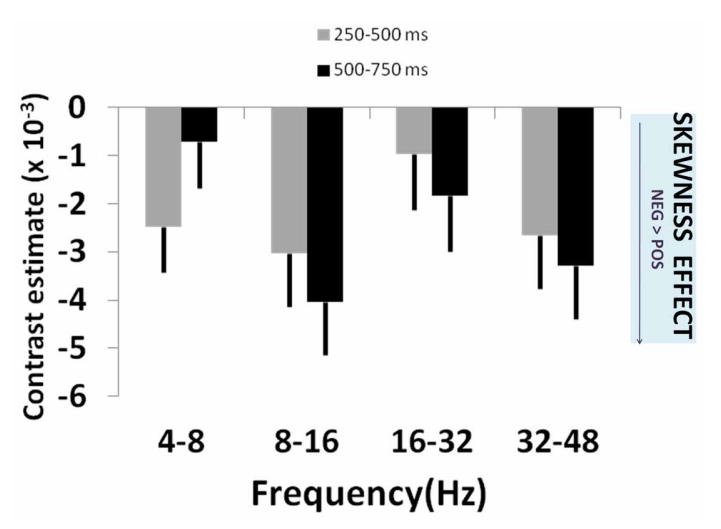

FIGURE 4 | Skewness effects across frequency bands. Plot of effect sizes for correlation of signal power with lottery skewness at 250-500 ms (gray bars) and 500-750 ms (black bars) time windows, for each of 4 specified frequency bands. These correspond to the time windows showing significant main effects in Figure 2. The uniformly negative contrast estimates reflect greater signal power for negatively skewed than positively skewed lotteries. See main text for details and statistical reporting.

\section{Induced responses to gamble versus sure choices}

fMRI studies have consistently reported differential activation during riskier or safer choices (Kuhnen and Knutson, 2005; Christopoulos et al., 2009; Xue et al., 2009; Symmonds et al., 2011), hence we next asked whether choice-specific modulations were evident in the MEG signal. We observed such modulation bilaterally over the central sulcus [right somatosensory 
cortex: $F_{(3,210)}=7.81 ; p=0.0001 ;$ left: $F_{(3,210)}=5.64, p=$ 0.001 ; Figure 2E], reaching a peak effect in the $750-1000 \mathrm{~ms}$ time window following stimulus presentation $\left[t_{(1,210)}=3.25\right.$, $p=0.001$; Figure $2 \mathrm{~F}$ ]. This choice-related activity peaked at a later time-point than signals corresponding to decision statistics, suggesting a sequence of cortical processing of statistical risk prior to formation of choice. This choice signal was expressed mainly as a reduction in $8-16$ and $32-48 \mathrm{~Hz}$ band power prior to choosing to gamble.

Exploring this further, we also asked whether choice-related signals were expressed uniformly across subjects, or whether risk-tolerant individuals show a different temporal profile to riskaverse individuals. We found a significant correlation between choice activity and both variance and skewness preference in anterior insula/inferior frontal gyrus (Figure 5A). This interaction of neural activity with behavioral preference was expressed just prior to decision execution in the 500-1000 $\mathrm{ms}$ window [conjunction analysis: $500-750 \mathrm{~ms}, t_{(1,54)}=2.69, p=0.005 ; 750-1000 \mathrm{~ms}$, $\left.t_{(1,54)}=2.75, p=0.004\right]$, but also in the first time window $\left[0-250\right.$ ms: variance coefficient $\times$ choice activity: $t_{(1,54)}=2.70$, $p=0.005$; Figure 5B $]$.

To illustrate these preference-dependent responses, we obtained a full time-frequency characterization using a pseudoinverse to extract regional signals from the anterior insula area displaying maximal effects of our parametric regressors. When we split subjects according to both variance and skewness preference, a general pattern emerged whereby variance tolerant, and positive-skew seeking individuals (tolerant of uncertainty

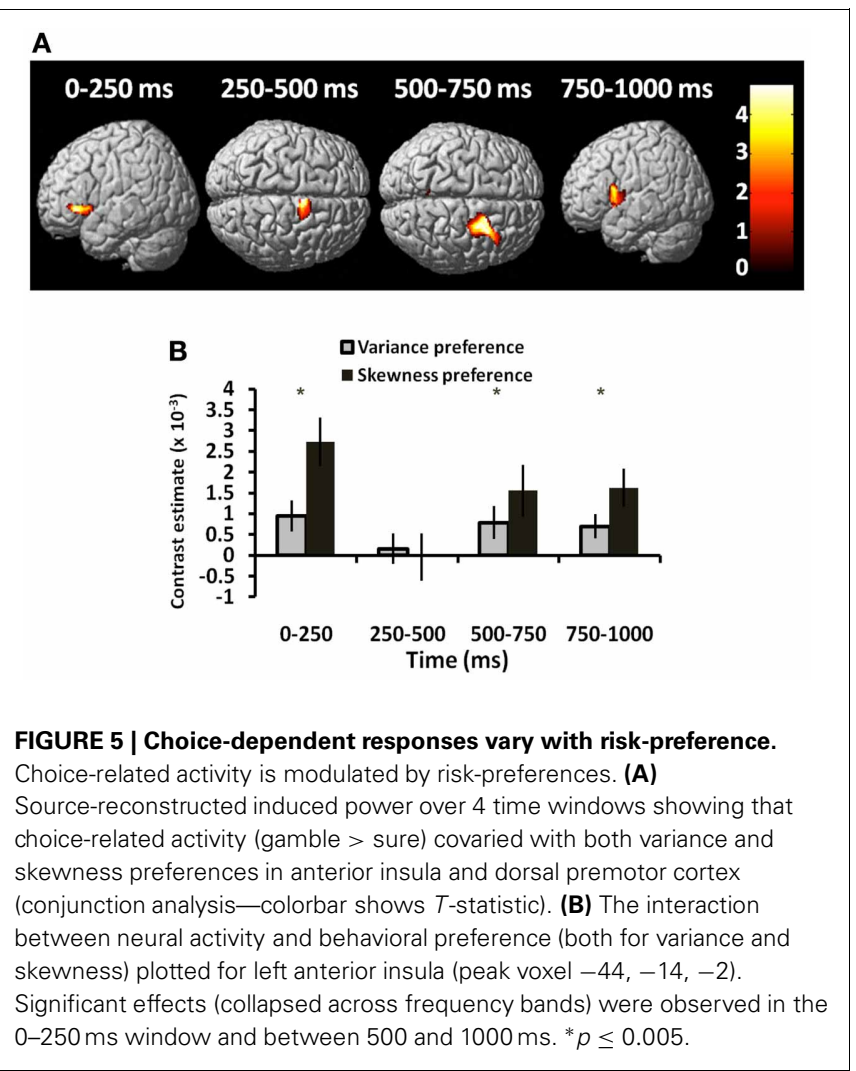

and driven by the potential of large rewards), express an early increase in power prior to a choice to gamble, while individuals with the opposite preference pattern exhibit enhanced activity much later (Figure 6). Thus, insula activity predicts gamble and sure choices, but exhibits a different time course of activity depending upon an individual's specific risk preferences. The early choice-predictive signal in insula occurs concurrently with encoding of variance and skewness in parietal and prefrontal cortices. Conversely, the late effect is consistent with an anticipatory response prior to action execution, with heightened activity prior to gamble choices in individuals who dislike taking risk.

\section{DISCUSSION}

In this study, we map the temporal evolution of induced responses to risk, separating effects of outcome dispersion or uncertainty (variance) and outcome asymmetry (skewness). We show these different aspects of statistical risk are processed as early as $250 \mathrm{~ms}$ in separate cortical regions, evident in changes of electromagnetic power in distinct frequency bands.

We suggest that early induced oscillatory activity during lottery evaluation represents encoding of relevant decision variables. These stimulus-locked responses are modulated, per trial, by both variance and skewness consistent with a highly specialized cortical evaluation of risk. By contrast, choice signals seen over the central

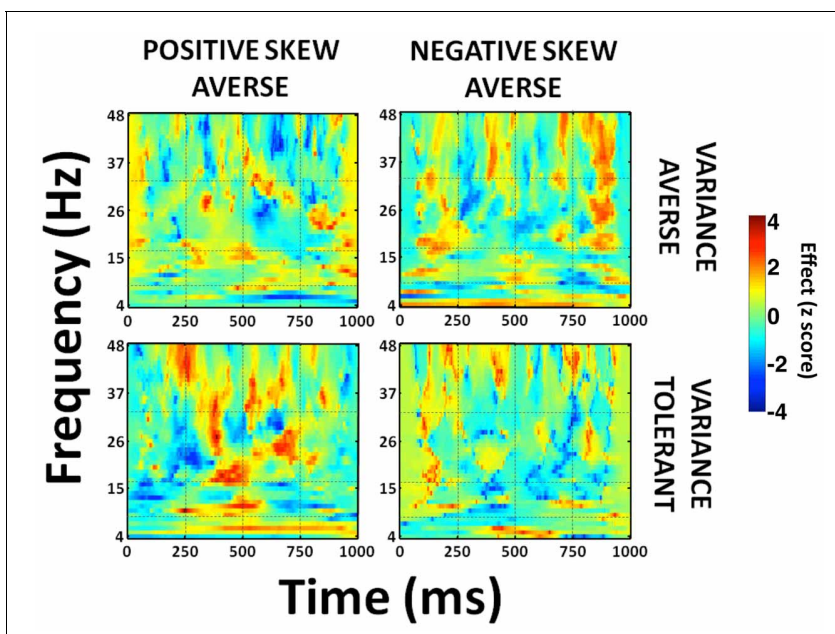

FIGURE 6 | Time-frequency plot of preference-dependent effects within anterior insula. Subjects were split into four groups-variance averse/tolerant and positive/negative skew averse individuals (median split). Full time-frequency characterization of the signals in the left anterior insula correlating with choice. This data extraction was performed in a $10 \mathrm{~mm}$ sphere centered on peak voxel coordinates (peak voxel MNI coordinates: $-44,-14,-2)$ from our statistical image using a source-extraction routine implemented in SPM. We used a Morlet wavelet time-frequency decomposition with 7 cycles, with a frequency-dependent cycle length as implemented in SPM8 (Kiebel et al., 2005). Gridlines show 4 time windows (0-250 ms, 250-500 ms, 500-750 ms, 750-1000 ms) and 4 frequency bands ["theta" $(4-8 \mathrm{~Hz})$, "alpha" $(8-16 \mathrm{~Hz})$, "beta" (16-32 Hz), "gamma" $(32-48 \mathrm{~Hz})]$. Late increased beta and gamma activity prior to gamble choices is most evident in variance and negative skew averse individuals, while early theta and beta activity prior to gamble choices is most evident in variance tolerant, positive skew averse individuals. Colorbar shows $z$ score values. $T=0$ corresponds to time of stimulus presentation. 
sulcus (i.e., sensorimotor cortex), occur after these risk encoding effects in parietal and prefrontal cortex, as indeed would be expected from the perspective of a process model of risk perception leading to subsequent evaluation and choice. Notably, these effects are expressed within previously identified risk-sensitive regions (Huettel et al., 2006; Platt and Huettel, 2008; Preuschoff et al., 2008; Mohr et al., 2010b). For example, a linear response to variance in parietal cortex occurs within the first $250 \mathrm{~ms}$, and in DMPFC to skewness, between 250 and $750 \mathrm{~ms}$ following stimulus presentation. This pattern supports behavioral evidence (Coombs, 1960; Coombs and Bowen, 1971; Peiro, 1999), and independently corroborates fMRI data (Burke and Tobler, 2011; Symmonds et al., 2011; Wu et al., 2011), that risk is not a unitary phenomenon but is a construct with independent dimensions that are each evaluated within discrete neural networks. PPC has been shown to accumulate perceptual evidence under uncertainty prior to action selection (Huk and Shadlen, 2005; Kiani et al., 2008), and has a general role in numerical and spatial quantification (Hubbard et al., 2005; Piazza et al., 2007) while DMPFC is implicated in encoding probability of loss (Smith et al., 2009; Xue et al., 2010), and is also consistently implicated in risk-processing (Tobler et al., 2007; Bach et al., 2009; Engelmann and Tamir, 2009; St. Onge and Floresco, 2009; Venkatraman et al., 2009; Mohr et al., 2010a). The distributed spatial processing of these risk dimensions corroborates an hypothesis that dispersion and relative hedonic asymmetry of outcomes are supported by separable, specialized, neural processing.

Although responses to risk $250 \mathrm{~ms}$ after stimulus onset are clearly rapid, these early responses are similar to processing speeds seen during perceptual decision-making, for example, judging direction in moving dot stereograms (Heekeren et al., 2006), complex discrimination (Fleming et al., 2010), or value comparisons (Milosavljevic et al., 2010; Steffen et al., 2011). Moreover, the parametric changes in induced oscillatory activity we observe are also found to underpin many cognitive processes including sensory perception (Gray and Singer, 1989; Tiitinen et al., 1993; Tallon-Baudry and Bertrand, 1999), comparison (Spitzer et al., 2010), and maintenance in working memory (Romo et al., 1999). It is important to note that individuals are unlikely to be performing an explicit computation of variance, rather a rapid assay of the spread of possible outcomes, for which variance is a surrogate marker (and highly correlated with other measures of dispersion in our stimulus set). Our data make the point that individuals independently respond to both dispersion and asymmetry of outcomes. These responses to variance and skewness overlap in time, although there is a suggestion that signals correlating with variance are detectable prior to those correlating with skewness. While this does not prove temporal precedence, it is interesting to note that this profile mirrors variance being a first-order measure of uncertainty while skewness (mathematically) reflects a secondorder attribute (the amount of relative gain vs. loss inherent in a gamble). Although recent investigations in trading behavior under time pressure suggest that skew sensitivity emerges rapidly (Nursimulu and Bossaerts, 2013) it is tempting to speculate that this time delay is sufficiently large to potentially affect outcomes in fast-paced markets (where orders can be transacted within $125 \mathrm{~ms})$.
Our findings where we map out frequency-based responses to risk are exploratory by nature given the dearth of prior research. While this makes it difficult to draw any conclusive inferences about the neurophysiological basis of the expressed effects in specific frequency bands, it is nevertheless notable that this frequency band profile differs for variance and skewness signals. For variance, we note an initial decrease in alpha band power at 0-250 ms. While early decreases in alpha have previously been observed in high-risk social decision-making situations (Qin et al., 2009; Billeke et al., 2012), decreased parietal alpha is also a marker of enhanced attention (Fries et al., 2001) and as all our subjects were variance-averse this relative decrement before selecting lower risk gambles could reflect a top-down attentional effect. This cannot explain the entire picture, and the cause of the subsequent broadband increase in power correlating with variance is less clear. Drawing a tentative parallel with similar cognitive processes, we note gamma band power increases in parietal lobe are associated with mathematical computation (Micheloyannis et al., 2005) but have not previously been shown to scale with specific stimulus attributes as we report here. This rebound increase in gamma signal power with high-risk gambles between 250 and $500 \mathrm{~ms}$ echoes findings in perceptual decision-making tasks requiring spatial judgments, where gamma-band enhancements are more evident for easier than more difficult decisions (Kaiser et al., 2007). Similarly, increased beta power has been shown in situations requiring a response inhibition (i.e., here, rejecting the gamble) (Cohen et al., 2008). Increased theta-band power is also seen at this timepoint, an effect that might be related to coupling between encoding and working memory in cross-cortical networks (Sauseng et al., 2005) in decision-making processes. Despite this, it is important to bear in mind that there is no one-to-one mapping between frequency bands and cognitive processes, and our speculations here are almost certainly over-simplistic.

The profile we observe for skewness is markedly different, with a unimodal change predominantly in alpha and gamma power. Increased gamma and alpha power are typically seen during evaluation of task-relevant variables and in maintenance of information in working memory (Tallon-Baudry and Bertrand, 1999; Jensen et al., 2007). Notably, there is greater right frontal alpha power for negatively than positively skewed gambles (i.e., situations with a small chance of a very poor outcome). Increased right frontal alpha power has been suggested to relate to avoidance or withdrawal and negative affect (Harmon-Jones et al., 2010). Skewness captures an implicit relative comparison of better than average to worse than average outcomes, hence we speculate that these alpha power changes may be involved in quantifying an asymmetry in valence of outcomes. Approach-avoidance processes are implicated in decision-making for gains and losses, and are distinct from processing of variance (Wright et al., 2012). However, it remains to be determined if skewness is encoded entirely independently of valence.

The choice signal was expressed mainly as a reduction in $8-16$ and $32-48 \mathrm{~Hz}$ band power prior to choosing to gamble. Reductions in $8-25 \mathrm{~Hz}$ band power (alpha/mu and beta band desynchronization-ERD), as well as a slow D.C. potential shift or magnetic field change (the "readiness potential") are typically 
observed prior to voluntary movement (Deecke et al., 1982; Cheyne, 2013). There is also evidence that this desynchronization during motor planning varies dependent upon the movement to be executed (Park et al., 2013), as well as cognitive variables such as response uncertainty (Tzagarakis et al., 2010). This lends further plausibility to our observations of differential signal patterns prior to gamble and sure responses, and the suggestion that these signals reflect specific motor preparation following lottery evaluation.

There is a tension in the theoretical literature between different models of decision-making under risk. Utility theory proposes risk sensitivity as an implicit by-product of a monotonically decreasing utility function (Pratt, 1964; Müller and Machina, 1987). In this schema, a separate processing pathway for risk is superfluous, as risk-preference is purely a consequence of translating objective quantities into subjective values. An alternative model, which has an affinity with financial theory and strongly supported by our data, suggests decisions involve an evaluation of the sufficient statistics or moments of a distribution of outcomes (expected value, variance and skewness). This is a useful heuristic in natural stochastic environments, where it is difficult to encode each possible outcome or state of the world with fidelity. The tracking of summary statistics is also efficient for learning, as it is computationally much easier to update these estimates rather than each outcome and its associated probability separately (D'Acremont and Bossaerts, 2008). While our paradigm is designed to be sensitive to risk effects rather than rule out a utility based encoding of value, our observation of early cortical responses to risk suggest that variance and skewness are relevant perceptual variables in the brain.

The insula has been implicated in risk-processing itself (Preuschoff et al., 2008), the integration of objective risk with subjective risk-preferences (Paulus et al., 2003; Christopoulos et al., 2009; Engelmann and Tamir, 2009; Xue et al., 2010), and in anticipating forthcoming risk (Kuhnen and Knutson, 2005). Intriguingly, we find the temporal profile of insula responses show two distinct effects, an early influence of risk-preference on choice-activity at $0-250 \mathrm{~ms}$ after stimulus presentation, and a later effect prior to making a decision at $750-1000 \mathrm{~ms}$. The early effect occurs concurrently with risk encoding in parietal and prefrontal cortices, indicating a possible role in a risk-processing network. The later insula response is consistent with an affective component in risky choice, particularly as it follows rather than precedes choice-sensitive premotor activity. We explored this further, partitioning anterior insula effects in individuals with different risk preference profiles to show positive skew seeking, variance tolerant individuals (similar to casino gamblers

\section{REFERENCES}

Akaike, H. (1974). A new look at the statistical model identification. IEEE Trans. Automat. Contr. 19, 716-723. doi: 10.1109/TAC.1974. 1100705

Bach, D., Seymour, B., and Dolan, R. (2009). Neural activity associated with the passive prediction of ambiguity and risk for aversive

who accept risk for the chance of high reward) show strong early responses before both gamble and sure choices rather than simply an early effect preceding a safe default option. More conservative individuals, preferring negative skewness and disliking uncertainty (variance), show dominance of late responses but only before choosing to gamble. Our data therefore hints at a dual role for insula, both at the time of risk encoding and also during entrainment of a motor program for action selection.

There are necessarily limitations to our study. MEG is maximally sensitive to cortical effects, hence we do not explore subcortical risk processing (Knutson et al., 2005; Preuschoff et al., 2006). However, this is compensated for by the fact that MEG allows resolution of risk-sensitive processes at sub-second timescales, measuring the temporal precedence of specific processes. This overcomes problems inherent in inferring causality from fMRI data (Friston, 2009). We focused on the chronometry of responses within the first second following stimulus presentation, as we were interested in processes underlying risk quantification and perception rather than responses locked to action execution. Importantly we show that signals correlating with statistical risk occur very early in decision-making, which strongly suggests a primary process of risk perception rather than evaluative responses emerging after the construction of actions.

\section{CONCLUSION}

Here we characterize the temporal sequence of neural responses during the perception and evaluation of risk. We show initial rapid processing of salient risk dimensions in PPC followed by DMPFC, manifest as parametric variations in electromagnetic responses correlating with these experimentally manipulated variables. We highlight a temporal dissociation in processing variance vs. skewness, and demonstrate that by mapping the sequence of neuronal activity at a sub-second timescale, it is possible to temporally dissect the dynamic processes that characterize risk evaluation. These early responses to independent statistical components indicate that specialized cortical circuitry underpins rapid decision-making under risk.

\section{ACKNOWLEDGMENTS}

This work was supported by the Wellcome Trust [Raymond J. Dolan Senior Investigator Award 098362/Z/12/Z]. The Wellcome Trust Centre for Neuroimaging is supported by core funding from the Wellcome Trust 091593/Z/10/Z. Rosalyn Moran is supported by an award from the Max Planck Society to Raymond J. Dolan; Nicholas D. Wright is supported by a Wellcome Trust Clinical Research Fellowship.

perception and predicts social decisions. Cereb. Cortex. doi: 10.1093/ cercor/bhs269. [Epub ahead of print].

Burke, C. J., and Tobler, P. N. (2011). Reward skewness coding in the insula independent of probability and loss. J. Neurophysiol. 106, 2415-2422. doi: 10.1152/jn.00471.2011
Cheyne, D. O. (2013). MEG studies of sensorimotor rhythms: a review. Exp. Neurol. 245, 27-39. doi: 10.1016/j.expneurol. 2012.08.030

Christopoulos, G., Tobler, P., Bossaerts, P., Dolan, R., and Schultz, W. (2009). Neural correlates of value, risk, and risk aversion contributing to decision making under risk. 
J. Neurosci. 29, 12574-12583. doi: 10.1523/JNEUROSCI.2614-09.2009

Clark, L., Bechara, A., Damasio, H., Aitken, M. R. F., Sahakian, B. J., and Robbins, T. W. (2008). Differential effects of insular and ventromedial prefrontal cortex lesions on risky decisionmaking. Brain 131, 1311-1322. doi: 10.1093/brain/awn066

Cohen, M. X., Elger, C. E., and Fell, J. (2008). Oscillatory activity and phase-amplitude coupling in the human medial frontal cortex during decision making. J. Cogn. Neurosci. 21, 390-402. doi: 10.1162/jocn.2008.21020

Coombs, C., and Bowen, J. (1971). A test of VE-theories of risk and the effect of the central limit theorem. Acta Psychol. 35, 15-28. doi: 10.1016/0001-6918(71)90028-X

Coombs, C. H. (1960). Components of risk in decision making: probability and variance preferences. J. Exp. Psychol. 60, 265-277. doi: 10.1037/h0041444

Critchley, H. D., Mathias, C. J., and Dolan, R. J. (2001). Neural activity in the human brain relating to uncertainty and arousal during anticipation. Neuron 29, 537-545. doi: 10.1016/S08966273(01)00225-2

D'Acremont, M., and Bossaerts, P. (2008). Neurobiological studies of risk assessment: a comparison of expected utility and meanvariance approaches. $\operatorname{Cog} n$. Affect. Behav. Neurosci. 8, 363 . doi: 10.3758/CABN.8.4.363

Deecke, L., Weinberg, H., and Brickett, P. (1982). Magnetic fields of the human brain accompanying voluntary movement: bereitschaftsmagnetfeld. Exp. Brain Res. 48, 144-148. doi: 10.1007/BF00239582

Engelmann, J., and Tamir, D. (2009). Individual differences in risk preference predict neural responses during financial decision-making. Brain Res. 1290, 28-51. doi: 10.1016/j.brainres.2009.06.078

Ernst, M., Bolla, K., Mouratidis, M., Contoreggi, C., Matochik, J. A., Kurian, V., et al. (2002). Decisionmaking in a risk-taking task: a PET study. Neuropsychopharmacology 26, 682-691. doi: 10.1016/S0893133X(01)00414-6

Fiorillo, C. D., Tobler P. N., and Schultz, W. (2003). Discrete coding of reward probability and uncertainty by dopamine neurons. Science 299, 1898-1902. doi: 10.1126/science. 1077349

Fleming, S. M., Whiteley, L., Hulme, O. J., Sahani, M., and Dolan, R. J. (2010). Effects of category-specific costs on neural systems for perceptual decision-making. J. Neurophysiol. 103, 3238-3247. doi: 10.1152/jn.01084.2009

Fries, P., Reynolds, J. H., Rorie, A. E., and Desimone, R. (2001). Modulation of oscillatory neuronal synchronization by selective visual attention. Science 291, 1560-1563. doi: $10.1126 /$ science. 1055465

Friston, K. (2009). Causal modelling and brain connectivity in functional magnetic resonance imaging. PLoS Biol. 7:e1000033. doi: 10.1371/journal.pbio. 1000033

Friston, K., Harrison, L., Daunizeau, J., Kiebel, S., Phillips, C., TrujilloBarreto, N., et al. (2008). Multiple sparse priors for the $\mathrm{M} / \mathrm{EEG}$ inverse problem. Neuroimage 39, 1104-1120. doi: 10.1016/j.neuroimage.2007.09.048

Gehring, W. J., and Willoughby, A. R. (2002). The medial frontal cortex and the rapid processing of monetary gains and losses. Science 295, 2279. doi: 10.1126/science. 1066893

Gray, C. M., and Singer, W. (1989). Stimulus-specific neuronal oscillations in orientation columns of cat visual cortex. Proc. Natl. Acad. Sci. U.S.A. 86, 1698. doi: 10.1073/pnas.86.5.1698

Harmon-Jones, E., Gable, P. A., and Peterson, C. K. (2010). The role of asymmetric frontal cortical activity in emotion-related phenomena: a review and update. Biol. Psychol. 84, 451-462. doi: 10.1016/j.biopsycho.2009.08.010

Harris, A., Adolphs, R., Camerer, C., and Rangel, A. (2011). Dynamic construction of stimulus values in the ventromedial prefrontal cortex. PLOS ONE 6:e21074. doi: 10.1371/journal.pone.0021074

Hedgcock, W., Crowe, D., Leuthold, A., and Georgopoulos, A. (2010). A magnetoencephalography study of choice bias. Exp. Brain Res. 202, 121-127. doi: 10.1007/s00221-0092117-6

Heekeren, H. R., Marrett, S., Ruff, D. A., Bandettini, P., and Ungerleider, L. G. (2006). Involvement of human left dorsolateral prefrontal cortex in perceptual decision making is independent of response modality. Proc. Natl. Acad. Sci.U.S.A. $103, \quad 10023-10028$. doi: $10.1073 /$ pnas. 0603949103

Hewig, J., Trippe, R., Hecht, H., Coles, M. G. H., Holroyd, C. B., and Miltner, W. H. R. (2007). Decision-making in blackjack: an electrophysiological analysis. Cereb. Cortex 17, 865-877. doi: 10.1093/cercor/bhk040
Hubbard, E., Piazza, M., Pinel, P., and Dehaene, S. (2005). Interactions between number and space in parietal cortex. Nat. Rev. Neurosci. 6, 435-448. doi: 10.1038/nrn1684

Huettel, S. A., Song, A. W., and McCarthy, G. (2005). Decisions under uncertainty: probabilistic context influences activation of prefrontal and parietal cortices. J. Neurosci. 25, 3304-3311. doi 10.1523/JNEUROSCI.5070-04.2005

Huettel, S. A., Stowe, C. J., Gordon, E. M., Warner, B. T., and Platt, M. L. (2006). Neural signatures of economic preferences for risk and ambiguity. Neuron 49, 765-775. doi: 10.1016/j.neuron. 2006.01.024

Huk, A. C., and Shadlen, M. N. (2005) Neural activity in macaque parietal cortex reflects temporal integration of visual motion signals during perceptual decision making. J. Neurosci. 25, 10420. doi 10.1523/JNEUROSCI.4684-04.2005

Jensen, O., Kaiser, J., and Lachaux, J. P. (2007). Human gammafrequency oscillations associated with attention and memory. Trends Neurosci. 30, 317-324. doi: 10.1016/j.tins.2007.05.001

Jullien, B., and Salanie, B. (2000). Estimating preferences under risk: the case of racetrack bettors. J. Polit. Econ. 108, 503-530. doi: $10.1086 / 262127$

Kaiser, J., Lennert, T., and Lutzenberger, W. (2007). Dynamics of oscillatory activity during auditory decision making. Cereb. Cortex 17, 2258-2267. doi: 10.1093/cercor/bhl134

Kiani, R., Hanks, T. D., and Shadlen, M. N. (2008). Bounded integration in parietal cortex underlies decisions even when viewing duration is dictated by the environment. J. Neurosci. 28, 3017. doi: 10.1523/JNEUROSCI.4761-07. 2008

Kiebel, S. J., Tallon Baudry, C., and Friston, K. J. (2005). Parametric analysis of oscillatory activity as measured with EEG/MEG. Hum. Brain Mapp. 26, 170-177. doi: 10.1002/hbm. 20153

Kim, S. G., Richter, W., and Uğurbil, K. (1997). Limitations of temporal resolution in functional MRI. Magn. Reson. Med. 37, 631-636. doi: 10.1002/mrm.1910370427

Knoch, D., Gianotti, L. R., PascualLeone, A., Treyer, V., Regard, M., Hohmann, M., et al. (2006). Disruption of right prefrontal cortex by low-frequency repetitive transcranial magnetic stimulation induces risk-taking behavior.
J. Neurosci. 26, 6469-6472. doi: 10.1523/JNEUROSCI.0804-06.2006

Knutson, B., Taylor, J., Kaufman, M., Peterson, R., and Glover, G. (2005). Distributed neural representation of expected value. J. Neurosci. 25, 4806-4812. doi: 10.1523/JNEUROSCI.0642-05.2005

Kuhnen, C. M., and Knutson, B. (2005) The neural basis of financial risk taking. Neuron 47, 763-770. doi: 10.1016/j.neuron.2005.08.008

Lancaster, J., Summerlin, J., Rainey, L., Freitas, C., and Fox, P. (1997). The Talairach Daemon, a database server for Talairach atlas labels. Neuroimage 5, S633.

Lancaster, J. L., Woldorff, M. G., Parsons, L. M., Liotti, M., Freitas, C. S., Rainey, L., et al. (2000). Automated Talairach atlas labels for functional brain mapping. Hum. Brain Mapp. 10, 120-131.

Litvak, V., and Friston, K. (2008). Electromagnetic source reconstruction for group studies. Neuroimage 42, 1490-1498. doi: 10.1016/j.neuroimage.2008.06.022

Maldjian, J. A., Laurienti, P. J., Kraft, R. A., and Burdette, J. H. (2003). An automated method for neuroanatomic and cytoarchitectonic atlas-based interrogation of fMRI data sets. Neuroimage 19, 1233-1239. doi: 10.1016/S1053-8119(03)00169-1

Mazziotta, J. (2001). A probabilistic atlas and reference system for the human brain: International Consortium for Brain Mapping (ICBM). Philos. Trans. R. Soc. B Biol. Sci. 356, 1293-1322. doi: 10.1098/rstb.2001.0915

McCoy, A. N., and Platt, M. L. (2005). Risk-sensitive neurons in macaque posterior cingulate cortex. Nat. Neurosci. 8, 1220-1227. doi: $10.1038 / \mathrm{nn} 1523$

Micheloyannis, S., Sakkalis, V. Vourkas, M., Stam, C. J., and Simos, P. G. (2005). Neural networks involved in mathematical thinking: evidence from linear and non-linear analysis of electroencephalographic activity. Neurosci. Lett. 373, 212-217. doi: 10.1016/j.neulet.2004.10.005

Milosavljevic, M., Malmaud, J., Huth, A., Koch, C., and Rangel, A. (2010). The drift diffusion model can account for the accuracy and reaction time of value-based choices under high and low time pressure. Judgm. Decis. Mak. 5, 437-449.

Mohr, P., Biele, G., and Heekeren, H. (2010a). Neural processing of risk. J. Neurosci. 30, 6613. doi: 10.1523/JNEUROSCI.0003-10.2010 
Mohr, P., Biele, G., Krugel, L., Li, S., and Heekeren, H. (2010b). Neural foundations of risk-return trade-off in investment decisions. Neuroimage 49, 2556-2563. doi: 10.1016/j.neuroimage.2009.10.060

Müller, S. M., and Machina, M. J. (1987). Moment preferences and polynomial utility. Econ. Lett. 23, 349-353. doi: 10.1016/0165-1765(87)90143-1

Nursimulu, A. D., and Bossaerts, P. (2013). Risk and reward preferences under time pressure. Rev. Financ. doi: 10.1093/rof/rft013

Park, H., Kim, J. S., and Chung, C. K. (2013). Differential beta-band event-related desynchronization during categorical action sequence planning. PLoS ONE 8:e59544. doi: 10.1371/journal.pone.0059544

Paulus, M. P., Rogalsky, C., Simmons, A., Feinstein, J. S., and Stein, M. B. (2003). Increased activation in the right insula during risk-taking decision making is related to harm avoidance and neuroticism. Neuroimage 19, 1439-1448. doi: 10.1016/S10538119(03)00251-9

Peiro, A. (1999). Skewness in financial returns. J. Bank. Financ. 23, 847-862. doi: 10.1016/S0378-4266(98)00119-8

Piazza, M., Pinel, P., Le Bihan, D., and Dehaene, S. (2007). A magnitude code common to numerosities and number symbols in human intraparietal cortex. Neuron 53, 293-305. doi: 10.1016/j.neuron. 2006.11.022

Platt, M. L., and Huettel, S. A. (2008). Risky business: the neuroeconomics of decision making under uncertainty. Nat. Neurosci. 11, 398-403. doi: $10.1038 / \mathrm{nn} 2062$

Pratt, J. W. (1964). Risk aversion in the small and in the large. Econometrica 32, 122-136. doi: 10.1093/rof/rft013

Preuschoff, K., Bossaerts, P., and Quartz, S. R. (2006). Neural differentiation of expected reward and risk in human subcortical structures. Neuron 51, 381-390. doi: 10.1016/j.neuron.2006.06.024

Preuschoff, K., Quartz, S. R., and Bossaerts, P. (2008). Human insula activation reflects risk prediction errors as well as risk. J. Neurosci. 28, 2745-2752. doi: 10.1523/JNEUROSCI.4286-07.2008

Qin, J., Lee, T., and Han, S. (2009). Theta and alpha oscillations linked to risk identifications.
Brain Res. 1269, 125-134. doi: 10.1016/j.brainres.2009.02.077

Romo, R., Brody, C. D., Hernández, A., and Lemus, L. (1999). Neuronal correlates of parametric working memory in the prefrontal cortex. Nature 399, 470-473. doi: 10.1038/20939

Sauseng, P., Klimesch, W., Schabus, M., and Doppelmayr, M. (2005) Fronto-parietal EEG coherence in theta and upper alpha reflect central executive functions of working memory. Int. J. Psychophysiol. 57, 97-103. doi: 10.1016/j.ijpsycho.2005.03.018

Schutter, D. J. L. G., de Haan, E. H. F., and van Honk, J. (2004). Anterior asymmetrical alpha activity predicts Iowa gambling performance: distinctly but reversed. Neuropsychologia 42, 939-943. doi: $\quad 10.1016 /$ j.neuropsychologia. 2003.11.014

Scott, R. C., and Horvath, P. A. (1980). On the direction of preference for moments of higher order than the variance. J. Financ. 35, 915-919. doi: 10.1111/j.15406261.1980.tb03509.x

Smith, B., Mitchell, D., Hardin, M., Jazbec, S., Fridberg, D., Blair, R., et al. (2009). Neural substrates of reward magnitude, probability, and risk during a wheel of fortune decision-making task. Neuroimage 44, 600-609. doi: 10.1016/j.neuroimage.2008.08.016

Spitzer, B., Wacker, E., and Blankenburg, F. (2010). Oscillatory correlates of vibrotactile frequency processing in human working memory. J. Neurosci. 30, 4496. doi: 10.1523/JNEUROSCI.6041-09.2010

Steffen, A., Rockstroh, B., Wienbruch, C., and Miller, G. A. (2011). Distinct cognitive mechanisms in a gambling task share neural mechanisms. Psychophysiology 48, 1037-1046. doi: $\quad 10.1111 / j .1469-8986.2011$. 01177.x

St. Onge, J., and Floresco, S. (2010). Prefrontal cortical contribution to risk-based decision making. Cereb. Cortex 20, 1816-1828.

St. Onge, J. R., and Floresco, S. B. (2009). Dopaminergic modulation of risk-based decision making. Neuropsychopharmacology 34, 681-697. doi: 10.1038/npp. 2008.121

Symmonds, M., Bossaerts, P., and Dolan, R. (2010). A behavioural and neural evaluation of prospective decision-making under risk.
J. Neurosci. 30, 14380-14389. doi: 10.1523/JNEUROSCI.1459-10. 2010

Symmonds, M., Wright, N., Bach, D., and Dolan, R. (2011) Deconstructing risk: separable encoding of variance and skewness in the brain. Neuroimage 58, 1139-1149. doi: 10.1016/j.neuroimage.2011.06.087

Tallon-Baudry, C., and Bertrand, O. (1999). Oscillatory gamma activity in humans and its role in object representation. Trends $\operatorname{Cog} n$. Sci. 3, 151-162. doi: 10.1016/S13646613(99)01299-1

Tiitinen, H., Sinkkonen, J., Reinikainen, K., Alho, K., Lavikainen J., and Näätänen, R. (1993). Selective attention enhances the auditory $40-\mathrm{Hz}$ transient response in humans. Nature 364 59-60. doi: 10.1038/364059a0

Tobler, P. N., O'Doherty, J. P., Dolan, R. J., and Schultz, W. (2007). Reward value coding distinct from risk attitude-related uncertainty coding in human reward systems. J. Neurophysiol. 97, 1621-1632. doi 10.1152/jn.00745.2006

Tobler, P. N., Christopoulos, G. I., O'Doherty, J. P., Dolan, R. J., and Schultz, W. (2009). Risk-dependent reward value signal in human prefrontal cortex. Proc. Natl. Acad. Sci. U.S.A. 106, 7185-7190. doi 10.1073/pnas.0809599106

Tzagarakis, C., Ince, N. F., Leuthold, A. C., and Pellizzer, G. (2010). Betaband activity during motor planning reflects response uncertainty J. Neurosci. 30, 11270-11277. doi 10.1523/JNEUROSCI.6026-09.2010

Venkatraman, V., Payne, J., Bettman, J., Luce, M., and Huettel, S. (2009). Separate neural mechanisms underlie choices and strategic preferences in risky decision making. Neuron 62, 593-602. doi: 10.1016/j.neuron.2009.04.007

Weber, E. U., and Johnson, E. J. (2008). "Decisions under uncertainty: Psychological, economic, and neuroeconomic explanations of risk preference," in Neuroeconomics: Decision making and the brain, eds P. Glimcher, C. Camerer, E. Fehr, and R. Poldrack (New York, NY: Elsevier), 127-144.

Wright, N. D., Symmonds, M. Hodgson, K., Fitzgerald, T. H. B., Crawford, B., and Dolan, R. J. (2012). Approach-avoidance processes contribute to dissociable impacts of risk and loss on choice. J. Neurosci. 32, 7009-7020. doi: 10.1523/JNEUROSCI.0049-12.2012

Wu, C. C., Bossaerts, P., and Knutson, B. (2011). The affective impact of financial skewness on neural activity and choice. PLoS ONE 6:e16838. doi: 10.1371/journal. pone. 0016838

Xue, G., Lu, Z., Levin, I., Weller, J., Li, X., and Bechara, A. (2009). Functional dissociations of risk and reward processing in the medial prefrontal cortex. Cereb. Cortex 19, 1019. doi: 10.1093/cercor/bhn147

Xue, G., Lu, Z., Levin, I. P., and Bechara, A. (2010). The impact of prior risk experiences on subsequent risky decisionmaking: the role of the insula. Neuroimage 50, 709-716. doi: 10.1016/j.neuroimage.2009.12.097

Yu, R., Zhou, W., and Zhou, X. (2011). Rapid processing of both reward probability and reward uncertainty in the human anterior cingulate cortex. PLOS ONE 6:e29633. doi: 10.1371/journal.pone.0029633

Conflict of Interest Statement: The authors declare that the research was conducted in the absence of any commercial or financial relationships that could be construed as a potential conflict of interest.

Received: 10 June 2013; paper pending published: 16 July 2013; accepted: 31 July 2013; published online: 20 August 2013. Citation: Symmonds M, Moran RJ, Wright ND, Bossaerts P, Barnes $G$ and Dolan RJ (2013) The chronometry of risk processing in the human cortex. Front. Neurosci. 7:146. doi: 10.3389/ fnins.2013.00146

This article was submitted to Decision Neuroscience, a section of the journal Frontiers in Neuroscience.

Copyright (C) 2013 Symmonds, Moran, Wright, Bossaerts, Barnes and Dolan. This is an open-access article distributed under the terms of the Creative Commons Attribution License (CC BY). The use, distribution or reproduction in other forums is permitted, provided the original author(s) or licensor are credited and that the original publication in this journal is cited, in accordance with accepted academic practice. No use, distribution or reproduction is permitted which does not comply with these terms. 